\title{
Clinical characteristics and relating factors of somatization symptoms of outpatients with psychological disorders: A cross-sectional observational study
}

\section{Shan Gong}

Xiangya Hospital Central South University

Yanan Zhang

Xiangya Hospital Central South University

Chao Jin

Xiangya Hospital Central South University

Liang Ning

Xiangya Hospital Central South University

Yi Xiao

Xiangya Hospital Central South University

Guolong Yu ( $\nabla$ 15576684661@163.com)

Xiangya Hospital Central South University

Research article

Keywords: general hospitals, outpatients, somatic symptoms, anxiety, depression.

Posted Date: September 23rd, 2019

DOI: https://doi.org/10.21203/rs.2.14740/v1

License: (c) (i) This work is licensed under a Creative Commons Attribution 4.0 International License.

Read Full License 


\section{Abstract}

Abstract Background:Cardiovascular disease patients associated with psychological disorders are very common clinically.It has been demonstrated in previous study that a higher prevalence of psychiatric disorders in the patients with CVD than the general population.Anxiety and depression are found to be the most common types of psychiatric disorders in the patients with CVD.

Objective:Exploring the clinical characteristics and relating factors of somatization symptoms of the outpatients with psychological disorders. Methods:From August 2017 to July 2018,we used the General Anxiety Disorder-7, Patient Health Questionnaire, Patient Health Questionnaire-15 and self-made general demographic data questionnaire to investigate the outpatients suspected with psychiatric disorders in cardiology department in a general hospital,who were screened with the"three questions"method.

Results:Among the 508 effective patients, the total detection rate of anxiety/depression was $81.1 \%$ $(412 / 508)$, and the detection rate of somatization symptoms was $93.1 \%(473 / 508)$. The positive number of PHQ-15 symptom items was $8.54 \pm 2.67$,and the detection rate of anxiety/depression was $78.7 \%$ in 473 patients with somatization symptoms.Among the patients with mild,moderate and severe somatization symptoms,there were significant differences in the proportion of women, the average number of the patient visits and hospitalizations in the past year $(P=0.0341,0.023)$, and also in GAD-7 and PHQ-9 scale scores $(P=0.0282,0.0011)$. Linear correlation analysis showed $P H Q-15$ score was significantly correlated with GAD-7 $(r=0.4787, P<0.001)$ and PHQ-9 score $(r=0.5141, P<0.001)$. Stepwise logistic regression analysis showed that the PHQ-9,GAD-7 scores and female were positive factors on somatizations.

Conclusion:The incidence of somatization symptoms is high in patients with psychological disorders in the outpatients of cardiology department.

\section{Background}

Cardiovascular disease(CVD) patients associated with psychological disorders are very common clinically. Compared with the general population, a higher prevalence of psychiatric disorders in the patients with CVD has been demonstrated in previous study[1,2]. Anxiety and depression are found to be the most common types of psychiatric disorders in the patients with CVD $[1,3]$. One recent research by Man Zhao et al, showed that the incidence rate of anxiety and depression was $42.5 \%$ in the outpatients of cardiology department of general hospitals[4]. Conversely, an association of psychiatric disorders, in particular anxiety and depression, with the development and progression of cardiovascular disease has been confirmed by recent research $[1,5,6]$. The common psychological problems such as depression and anxiety are often accompanied by somatic complaints including chest pain, fatigue, shortness of breath, dizziness, etc.[7-10]. Patients lacking insight into psychological problems often consult general practitioners or physical medicine specialists regarding such somatic symptoms[7-9]. However, the practitioners in general hospitals have limited ability to recognize and deal with psychological disorders [11]. As a result, persistent somatic symptoms are associated with high medical and societal costs and 
unmet clinical need[12]. Therefore, it is very important for clinicians to strengthen the identification and intervention of psychological problems in general hospitals. To our knowledge, the clinical research on somatization symptoms is rare in the outpatients with CVD in general hospitals.

This present study was aimed to investigate the clinical features and influencing factors of somatization symptoms of the outpatients suspected with psychiatric disorders in Department of Cardiology in a tertiary general hospital, who complained of physical discomfort and was considered as a suspicious psychological disorder by screened with the "Three Questions" method recommended by the Chinese Expert Consensus on Psychological Prescriptions of Cardiovascular Patients in 2014[13].

\section{Methods}

\subsection{Participants}

We recruited the outpatients who complained of physical discomfort and visited the Department of Cardiology at the Xiangya Hospital, Central South University, Changsha, Hunan consecutively from August 2017 to July 2018. Patients who met the both of following criteria were included:1.The both of "three questions "were positive in the "three questions method"in the patient, who was asked to take part in a screening method with the "three questions method" [13] and considered as a suspicious psychological disorder. 2.Patients over 18 years old who were willing to cooperate and were able to complete the scales and questionnaires. Exclusion criteria included:severe hypertension, heart failure above New York Heart Association(NYHA) grade III, severe arrhythmias such as ventricular tachycardia and atrial fibrillation; somatic diseases such as diabetes mellitus, severe organic brain diseases, dementia and cognitive impairment, hepatic and renal insufficiency, respiratory failure; difficulties in communication, language or writing; schizophrenic psychiatric symptoms and severe depression with suicidal tendencies and behaviors; and having taken or being taking related drugs (such as anti-anxiety and depressive drugs, sedative and hypnotic drugs, analgesic drugs, etc.) after the onset of physical symptoms.

\subsection{Methods}

Participants were assisted to complete the following information assessment by the coordinators with certificate of second-level psychological counselor who received unified training:

General demographic data:it includes age, gender, marital, status, education, occupation, current disease diagnosed by clinical and electrocardiogram, echocardiogram and other auxiliary examinations, duration of the disease, as well as the medical consultation and treatment for main somatization symptoms in the past one year.

And Table 1 showed other three scales:Anxiety Disorder-7(GAD-7) $14 \rrbracket$, Chinese version of The 9symptom Patient Health Questionnaire (PHQ-9) $15 \mathbb{1 5}$,Chinese version of The 15-symptom Patient Health 


\subsection{Statistical methods}

All data were analyzed with SPSS Version 17.0 (IBM, Armonk, NY). The measurement data of normal distribution were expressed as mean \pm SD. The counting Data were described with rank or percentage. According to the data types and distribution characteristics, one-way ANOVA was used to compare the mean between groups, $\mathrm{X}^{2}$ test used to compare the categorical data and ratios. Linear correlation analysis was adopted for the two-variable relationship, and stepwise logistic regression analysis with the forward conditional method was used for the multi-factor analysis. Bilateral test $P<0.05$ was considered statistically significant.

\section{Results}

\section{The occurrence of anxiety/depression and somatic symptoms}

538 patients suspected with psychological disorders were found by screened with the"three questions method"[13]. Among them, 30 refused to complete the scales and Questionnaires because of lack of time, unwillingness to disclose personal data and rejection to the self-rated psychological scale. 508 consecutive eligible adult outpatients completed all questionnaires and were recruited in this study. The study protocol was approved by the Ethics Committees of Xiangya Hospital, Central South University and informed consent was obtained from all of the participants. Among the 508 patients, $316(62.2 \%)$ of which were women, age ranged from 18 to 82 , with mean age of $51.3 \pm 10.1$ years old. A total of 278 $(54.72 \%)$ were diagnosed as cardiovascular diseases, including 138 with hypertension, 30 with unstable angina pectoris, 24 with stable angina pectoris, 56 with arrhythmia such as frequent ventricular premature, frequent atrial premature and short paroxysmal atrial tachycardia; 129 (25.39\%) were diagnosed as other somatic diseases, including 56 with chronic gastritis, 34 with multiple lacunar infarction of brain and 36 with cervical spondylosis and other diseases, and 102 cases $(20.07 \%)$ having no somatic diseases.

Among the 508 patients, anxiety symptoms (GAD-7 $\geq 5$ ) were found in 359 patients $(70.7 \%)$ and depression symptoms (PHQ-9 $\geq 5$ ) in 387 patients(76.2\%). Depression coexisted with anxiety symptoms were detected in 305 patients $(60.0 \%)$, and the total number of patients with anxiety or depression symptoms reached to 412 cases (81.1\%). Somatization symptoms (PHQ-15 $\geq 5$ ) were identified in 473 patients(93.1\%).

Among all the patients with somatization symptoms, the positive number of the items in PHQ-15 was $8.04 \pm 2.67$, and the total number of patients associated with anxiety or depression symptoms was 400 $\operatorname{cases}(78.7 \%)$. 


\subsection{Distribution characteristics of positive number of PHQ- 15 in patients with somatization symptoms}

Table 2 showed that the total number of positive PHQ-15 symptoms items in 473 patients with somatization symptoms was 3168, of which 2159 positive items with score $=1$ and 1009 items with score $=2$. Among the positive PHQ-15 symptoms, more than $50 \%$ of the positive symptoms were sleep disorder, chest pain, headache, dyspnea, palpitation, dizziness and nausea, flatulence or dyspepsia, while less than $10 \%$ were menstrual problems or cramps, sexual pain or dysfunction.

\subsection{Comparison of general demographic data in patients with different degrees of somatization symptoms}

Based upon the sum of somatization symptom items, participants were categorized into 3 groups:154 (32.6\%) with mild (5-9), 251 (53.07\%) with moderate (10-14), and 68 (14.4\%) with severe ( $\geq 15)$.

The table 3 showed the characters of patients with somatization symptoms:compared among the three groups of patients with different degrees of somatic symptoms, there was no significant difference in the average age, marital status, education level, occupation, the duration of illness and the proportion of current diseases, but significant difference was found in the average total score of PHQ-15, the proportion of women, and the average number of outpatient visits and hospitalizations due to the main somatic symptoms within the past one year.

\subsection{Comparison of anxiety and depression scores in patients with different degrees of somatization symptoms and analysis of correlation between PHQ-15 score and anxiety or depression}

As observed in table 4, both anxiety and depression scores increased successively in the patients with somatization symptoms of mild, moderate and severe degrees, and there were statistically significant differences between the groups with different degrees of somatic symptoms. The correlation analysis showed that the correlation coefficient between PHQ-15 and GAD-7 scores in the patients with somatization symptoms was 0.4787 , with statistical significance $(P<0.001)$; and the correlation coefficient between PHQ-15 and PHQ-9 scores was 0.5141 , with statistical significance $(P<0.001)$.

\subsection{Analysis of predictive factors for somatic symptoms in the patients}


Stepwise Logistic regression analysis was performed to analyze the predictive factors for somatization symptoms. Factors with significant differences (total score of PHQ-9 and GAD-7) and demographic factors (such as female) were included into the predictive factors for stepwise Logistic regression analysis. Table 5 showed that the score of PHQ -9 and GAD- 7 and the female were positive predictive factors for somatization symptoms. The probability of somatization increased by 6.526 and 8.02 times respectively for each 1 point increase in $G A D-7$ and PHQ -9 . The likelihood of female suffering from somatization symptoms increased by 4.440 times. The results indicated that depression, anxiety and female were the predictors of somatic symptoms.

\section{Discussion}

The somatization symptoms of patients in general hospitals have become more and more serious clinical and public health problems[17]. Somatization symptoms[18] refers to the experience and presentation of somatic discomfort, which cannot be explained by the knowledge or experience have known.In clinic,disease-related somatic symptoms are different from somatization symptoms, which can be explained by corresponding diseases, and have disease-related clinical characteristics.

Somatization symptoms are quite prevalent in the patients in general hospitals[11,17]. Patients often lack awareness of psychological problems and consider that somatization symptoms are caused by somatic diseases, so they tend to frequently seek the medical service,even visit a tertiary hospital directly. Anxiety and depression are common psychological disorders in outpatients of Cardiology department[4], which are often manifested by various prominent symptoms of cardiovascular somatization, such as "heart distress" or "racing heart". One recent report by Ye Ruifen[19], found that the incidence of somatization symptoms in outpatients of cardiology was as high as $64.2 \%$.

The present study was different from the published reports. Firstly, the object in the study was not all outpatients in cardiology department, but the ones suspected with psychological disorders, screened by the "three questions"[13], were selected as object in the study. Secondly, the three Chinese versions of GAD-7, PHQ-9 and PHQ-15 recommended by DSM-5 were used as research tools in this study. Several previous studies[16, 20,21] confirmed that the three scales could be used to screen anxiety, depression and somatization symptoms and assess the severity of anxiety, depression and somatization with good validity and reliability respectively in general hospitals in China.

The results of this study showed that the total detection rate of anxiety/depression and somatization symptoms was $81.1 \%$ and $93.1 \%$ respectively in the participants. This finding further confirmed that the "three questions" method[13] had a high validity for the initial screening of psychological disorders. This study also suggested that the incidence of somatization symptoms in the patients screened by "three questions" method[13] is much higher than that of $64.2 \%$ in the total outpatients in cardiology clinics [19].

This study found the average number of positive items of symptoms in PHQ-15 was 8 more than in the patients with somatization symptoms. Observing from the positive items of $\mathrm{PHQ}-15$ symptoms, the common items are ones related with cardiovascular diseases such as chest pain, palpitation, dyspnea. 
The positive items also included ones with nervous system such as headache, dizziness, and ones with digestive system such as gastralgia, abdominal distention.It was worth noting that the incidence of sleep disorder in PHQ-15 items was as high as 77.38\%, which indicates that sleep disorder was an important clinical feature of somatization symptoms in outpatients with psychological disorders in cardiology department.Item 4 (menstrual problems), and item 8 (sexual problems) displayed extreme floor effects. These floor effects were also found in previous Chinese and Western studies[22, 23], which may be related to the influence of Chinese traditional culture and the patients' lacking attention or unwillingness to express.

The results showed that anxiety and depression in patients with somatization symptoms were mild to moderate, and not all patients with somatization symptoms could meet the criteria of anxiety and depression by GAD-7 and PHQ-9 scales. In this study, the total detection rate of anxiety/depression in the patients with somatization symptoms was only $78.7 \%$, whereas $21.3 \%$ of the patients were negative.

The reason for negative results of anxiety/depression test in the patients with somatization symptoms[24, 25] may be as follows:(1)Anxiety/Depression Scale is compiled by psychologist, and its items mainly reflect psychological mood and symptoms; (2)patients often suffer from mild or moderate psychological disorders and are mainly concerned about their somatization symptoms; (3)Because of many factors including the stigma, patients often have a denial and exclusion attitude towards psychological and emotional problems, even if they have psychological and emotional problems, they will also neglect it. The study by Greden'[26] suggested that $69 \%$ of depressive patients complained only of somatic symptoms. Even when asked directly, $11 \%$ of them denied having any psychological symptoms of depression.

A lot of recent reports $[17,19,27]$ on the occurrence and influencing factors of somatization symptoms in outpatients of general hospitals has confirmed that somatization symptoms are closely related to anxiety and depression. In the present study, the results showed that the scores of GAD-7 and PHQ-9 increased with the degree of somatization symptoms, and there were significant differences among different degrees of somatization symptoms in GAD-7 and PHQ-9 scores. There were no significant differences in average age, marital status, education level, occupation, duration of illness and the proportion of current illness except for the proportion of women in different degrees of somatization symptoms. Stepwise logistic regression analysis also found that the total scores of PHQ-9 and GAD-7 and female were the positive correlative factors of somatization symptoms. The results of our study were consistent with the relevant researches[17, 19,27], it further reveals that anxiety, depression and gender are the main predictive factors for somatization symptoms of outpatients in cardiology department of general hospitals. The correlation mechanism between somatization symptoms and anxiety and depression[28, 29] may be as follows:(1)Depression and anxiety are the most common direct causes of somatization, that is, patients mainly adopt somatic response rather than cognitive response when they are activated by mental stimulation. Somatization symptoms can be considered as the manifestation of anxiety and depression. (2)Depression and anxiety are the inducing or triggered factors of somatic diseases, such as hypertension and coronary heart disease. (3)Anxiety and depression may be a direct consequence of 
somatic diseases. (4)Anxiety and depression may aggravate the symptoms associated with physical diseases. Besides, the female is the influencing factor of somatization symptoms, and it may be related to the character of female, social and family status, and Chinese cultural traditions.

The present study has several limitations. First, this study was a single-center cross-sectional study. Our study sample consisted of the patients who visit the cardiology department in a tertiary hospitals and were initially screened as psychological disorders. Therefore, the results may be representative of somatization symptoms of patients suspected with psychological disorders. Second, the diagnosis of somatic diseases in a small number of patients was based on the patient's statement without confirmed by corresponding objective examinations. Finally, most of the patients were self-paid because of the medical insurance, and some patients were reluctant to disclose their personal or family financial situation when they completed general information. Therefore, this study did not include the economic status and means of payment of medical cost. One recent study by Xu W, et al,[30] found that whether outpatient expenses could be reimbursed was one of the influencing factors for somatization symptoms.

\section{Conclusion}

In conclusion, the incidence of somatization symptoms is high in patients with psychological disorders in outpatients of cardiology department of general hospitals. Anxiety, depression and gender are the main affecting factors on somatization symptoms. Early recognition and correct diagnosis of somatization symptoms in the patients with psychological disorders could lead to appropriate treatment and health care cost reduction. These results may help cardiologists in the general hospitals to diagnose and manage patients with psychological disorders appropriately.

\section{Abbreviations}

CVD:cardiovascular disease

DSM-5:Diagnostic of Mental Disorders, 5th edition GAD-7:General Anxiety Disorder-7

NYHA:New York Heart Association

PHQ-9:9-symptom Patient Health Questionnaire

PHQ-15:15-symptom Patient Health Questionnaire

\section{Declarations}

\section{Ethics approval and consent to participate}


This trail was approved by the Ethics Committee of Xiangya Hospital,Central South University. All participants in this study have been informed and verbal consent, for no personal information of participants was disclosed in this article.

\section{Consent for publication}

The manuscript has been approved by all the authors for publication in your journal.

\section{Availability of data and material}

Not applicable.

\section{Competing Interests}

The authors declare no conflict of interest.

\section{Acknowledgement}

And grateful acknowledgement is made to our supervisor Mr. Guolong Yu who gave us considerable help by means of suggestion, comments and criticism. In addition, we deeply appreciate the contribution to this thesis made in various ways by our friends and classmates.

\section{Funding}

This work was supported by Xiangya Hospital of Central South University.

\section{Authors Contributions}

SG and YZ contribute equally as first authors. CJ,LN,YX all participated in data collection, collation and discussion.SG and $Y Z$ were responsible for the original draft. Our tutor, professor GY, provided suggestions for the final draft.

\section{References}

1. Annagür, BB., Avci, A., Demir, K., \& Uygur, Ö. F. Is there any difference between the early age myocardial infarction and late age myocardial infarction in terms of psychiatric morbidity in patients who have survived acute myocardial infarction[J]. Comprehensive Psychiatry, 2015; 57, 10-15. 
2. Li Z, Li Y, Chen L. Prevalence of Depression in Patients With Hypertension: A Systematic Review and Meta-Analysis. Medicine (Baltimore) [J]. 2015; 94(31):e1317.

3. Albus C. Psychological and social factors in coronary heart disease, Ann. Med. 2010; 42(7):487-494.

4. Zhao M,Yu GL,Yang TL. Investigate the incidence of anxiety and depression in outpatients from cardiovascular department in a general hospital[J]. Chinese Journal of Clinical Psychology, 2012, 20(2):188-189. (in chinese).

5. Kollia N., Panagiotakos DB., Georgousopoulou E., et al. Exploring the association between low socioeconomic status and cardiovascular disease risk in healthy Greeks, in the years of financial crisis (2002-2012):the ATTICA study[J]. Int. J. Cardiol. 2016:223 (15): 758-763.

6. Tully PJ., Turnbull DA., Beltrame J., Horowitz J., Cosh S., Baumeister H., et al., Panic disorder and incident coronary heart disease: a systematic review and metaregression in 1131612 persons and 58111 cardiac events[J]. Psychol. Med. 2015; 45(14):2909-2920.

7. Koh DJ, Kim NY, Kim Y. Predictors of Depressive Mood in Patients With Isolated Cerebellar Stroke: A Retrospective Study[J]. Ann Rehabil Med. 2016; 40(3):412-419.

8. Kohlmann S1, Gierk B, Hümmelgen M, Blankenberg S, Löwe B. Somatic symptoms in patients with coronary heart disease:prevalence, risk factors, and quality of life[J]. JAMA Intern Med. 2013;173(15):1469-71.

9. Wu H, Zhao X, Fritzsche K, Salm F, Leonhart R, Jing W. Negative illness perceptions associated with low mental and physical health status in general hospital outpatients in China[J]. Psychol Health Med. 2014; 19(3):273-85.

10.Haftgoli N, Favrat B, Verdon F, Vaucher P, Bischoff T, Burnand B.Patients presenting with somatic complaints in general practice: depression, anxiety and somatoform disorders are frequent and associated with psychosocial stressors[J]. BMC Fam Pract. 2010; 11(1):1-8.

11.Zhu C, Ou L, Geng Q, Zhang M, Ye R, Chen J. Association of somatic symptoms with depression and anxiety in clinical patients of general hospitals in Guangzhou, China[J]. Gen Hosp Psychiatry. 2012; 34(2):113-20.

12.Wang J, Wang Q, Wimalaratne I. Chinese non-psychiatric hospital doctors' attitudes toward management of psychological/psychiatric problems[J]. BMC Health Serv Res. 2017;22; 17(1):576584.

13.Chinese society of rehabilitation cardiovascular disease professional committee,China geriatrics society of cardiovascular and cerebrovascular diseases professional committee. China expert consensus on the psychological prescription of cardiovascular patients[J]. Chinese Journal of Cardiology, 2014; 42(1):6-13.[in Chinese].

14.Spitzer RL, Kroenke K, Williams JB, Lowe B. A brief measure for assessing generalized anxiety disorder: the GAD-7[J]. Arch Intern Med.2006 166(10): 1092-1097. 
15.Kroenke K, Spitzer RL, Williams JB.The PHQ-9: validity of a brief depression severity measure[J]. J Gen Intern Med.2001;16(9): 606-613.

16.Zhang L, Fritzsche K, Liu Y, Wang J, Huang M, Wang Y. Validation of the Chinese version of the PHQ-15 in a tertiary hospital[J]. BMC Psychiatry, 2016;16(5): 89.

17.Ishikawa Y, Takeshima T, Mise J. Physical symptoms in outpatients with psychiatric disorders consulting the general internal medicine division at a Japanese university hospital[J]. Int J Gen Med. 2015:13; 8:261 -266.

18.Isaac ML, Paauw DS. Medically unexplained symptoms[J]. Med Clin North Am. 2014 May;98(3):663-672.

19.Ye RF, Geng QS, Qu LM. Correlative analysis between anxiety, depression and somatic symptoms in outpatients in cardiology clinic[J]. Lingnan Journal of Cardiovascular Disease, 2013(19):48-51.

20.Liao SC, Huang WL, Ma HM. The relation between the patient health questionnaire-15 and DSM somatic diagnoses. BMC Psychiatry. 2016;16(1):351-359.

21.Wang J, Guo WJ, Mo LL. Prevalence and strong association of high somatic symptom severity with depression and anxiety in a Chinese inpatient population[J]. Asia Pac Psychiatry. 2017 Dec;9(4). doi: 10.1111/appy.12282.

22.Qian J, Ren ZQ, Yu DH. The value of the Patient Health Questionnaire-15 (PHQ-15) for screening somatic symptoms in general hospital[J]. Chin Ment Health J. 2014;28:173-178.

23.Körber S, Frieser D, Steinbrecher N, Hiller W. Classification characteristics of the Patient Health Questionnaire-15 for screening somatoform disorders in a primary care setting[J]. J Psychosom Res. 2011;71:142-147.

24.Löwe B1, Spitzer RL, Williams JB. Depression, anxiety and somatization in primary care: syndrome overlap and functional impairment[J]. Gen Hosp Psychiatry. 2008;30(3):191-199.

25.Lieb R1, Meinlschmidt G, Araya R. Epidemiology of the association between somatoform disorders and anxiety and depressive disorders: an update[J]. Psychosom Med. 2007;69(9):860-863.

26.Greden JF. Physical symptoms of depression:unmet need[J].J Clin Psychiatry, 2003,64 (suppl7):5-11.

27.Zhou X, Min S, Sun J. Extending a structural model of somatization to South Koreans: Cultural values,somatizationtendency, and the presentation of depressive symptoms[J]. J Affect Disord. 2015;176(1):151-154. 
28.Aamland A, Malterud K, Werner EL. Patients with persistent medically unexplained physical symptoms: a descriptive study from Norwegian general practice[J].BMC Fam Pract. 2014; (29) 15:107-117.

29.Shraim M, Mallen CD, Dunn KM. GP consultations for medically unexplained physical symptoms in parents and their children: a systematic review[J]. Br J Gen Pract. 2013;63(610):e318-325.

30.Xu W, Liu JS, Mao JL. Investigation and analysis of multiple somatic symptoms of Chinese expatriates in general and internal medicine outpatient departments of foreign-funded hospitals[J]. Clin J Med Offic, 2016;44(3):304-308.[in Chinese].

\section{Tables}

Table 1: Scales the participants completed 
Generalized Anxiety Disorder-

7 (GAD-7)

Patient Health Questionnaire-9

(PHQ-9)

15-symptom Patient Health

Questionnaire (PHQ-15)
7 items, 4-point(0-3) response

Likert scare $(0=$ not at all to $3=$ nearly every day)

9 items,4-point(0-3) response

Likert scare $(0=$ not at all to $3=$ nearly every day)

15 items,3-point(0-2) response

Likert scale $(0=$ not bothered at all to $2=$ bothered a lot)
Anxiety

levels:

minimal $(0-$

4),

minimal $(0-$

4),

moderate

(10-14),

severe $(\geq 15)$.

Depression

levels:

minimal (0-

4),

mild (5-9),

moderate

(10-14),

severe $(\geq 15)$.

somatization

levels:

minimal (0-

4),

mild (5-9), 
moderate

$(10-14)$,

moderate

$(10-14)$.

Table 2: Prevalence of Somatic Symptoms of PHQ-15 in Patients with Somatization Symptoms 


\begin{tabular}{|c|c|c|c|}
\hline Somatic Symptoms & $\begin{array}{c}\text { Bothered a Little }] \\
\text { Score }=1\end{array}$ & $\begin{array}{c}\text { Bothered a Lot, } \\
\text { Score }=2\end{array}$ & $\begin{array}{c}\text { Total Positive } \\
\text { Rate }\end{array}$ \\
\hline Stomach pain & 150 & 85 & $49.68 \%$ \\
\hline Back pain & 133 & 79 & $44.82 \%$ \\
\hline Pain in arms, legs or joints & 88 & 59 & $31.08 \%$ \\
\hline $\begin{array}{c}\text { Menstrual problems or } \\
\text { cramps* }\end{array}$ & 21 & 5 & $5.50 \%$ \\
\hline Headaches & 224 & 97 & $67.86 \%$ \\
\hline Chest pain & 249 & 111 & $76.11 \%$ \\
\hline Dizziness & 158 & 82 & $50.74 \%$ \\
\hline Fainting spells & 48 & 29 & $16.28 \%$ \\
\hline $\begin{array}{c}\text { Feeling your heart pound or } \\
\text { race }\end{array}$ & 199 & 98 & $62.79 \%$ \\
\hline Shortness of breath & 221 & 85 & $64.69 \%$ \\
\hline $\begin{array}{l}\text { Pain during sexual } \\
\text { intercourse }\end{array}$ & 19 & 3 & $4.65 \%$ \\
\hline Constipation, diarrhea & 81 & 38 & $25.16 \%$ \\
\hline $\begin{array}{c}\text { Nausea, flatulence or } \\
\text { dyspepsia }\end{array}$ & 194 & 45 & $50.53 \%$ \\
\hline $\begin{array}{c}\text { Feeling tired or having low } \\
\text { energy }\end{array}$ & 123 & 78 & $42.49 \%$ \\
\hline Trouble sleeping & 251 & 115 & $77.38 \%$ \\
\hline $\begin{array}{c}\text { Number of positive symptom } \\
\text { items }\end{array}$ & 2159 & 1009 & \\
\hline
\end{tabular}

*ロOnly women were asked about menstrual symptoms

Table 3:Characters of patients with somatization symptoms 


\begin{tabular}{|c|c|c|c|c|c|}
\hline & $\begin{array}{c}\text { Mild } \\
(\mathrm{n}=154)\end{array}$ & $\begin{array}{l}\text { Moderate } \\
(\mathrm{n}=251)\end{array}$ & $\begin{array}{l}\text { Severe } \\
(\mathrm{n}=68)\end{array}$ & $\mathbf{F}$ & $\begin{array}{c}P \\
\text { Value }\end{array}$ \\
\hline PHQ-15 score & $6.89(2.11)$ & $12.43(1.52)$ & $17.66(3.06)$ & & 0.002 \\
\hline Age, years & $52.5(11.2)$ & $50.2(9.5)$ & $49.7(10.3)$ & & 0.442 \\
\hline Female $₫ \% \square$ & $89(57.8)$ & $151(60.2)$ & $50(73.5)$ & & 0.034 \\
\hline \multicolumn{6}{|l|}{ Education level(\%) } \\
\hline Primary school or below & $53(34.4)$ & $95(37.8)$ & $27(39.7)$ & & \multirow[t]{3}{*}{0.708} \\
\hline Middle school & $72(46.8)$ & $113(45.0)$ & $29(42.6)$ & & \\
\hline College degree or above & $29(18.8)$ & $43(17.2)$ & $12(17.7)$ & & \\
\hline \multicolumn{6}{|l|}{ Marital status(\%) } \\
\hline Married & $133(86.4)$ & $222(88.4)$ & $56(82.4)$ & & \multirow[t]{3}{*}{0.634} \\
\hline Unmarried & $15(9.7)$ & $20(8.0)$ & $8(11.8)$ & & \\
\hline Divorce or widow & $6(3.9)$ & $9(3.6)$ & $4(5.8)$ & & \\
\hline \multicolumn{6}{|l|}{ Occupation(\%) } \\
\hline Employed & $54(35.1)$ & $68(27.1)$ & $22(32.4)$ & & \multirow[t]{3}{*}{0.264} \\
\hline Unemployed or student & $72(46.8)$ & $131(52.2)$ & $34(50.0)$ & & \\
\hline Retired & $28(18.1)$ & $52(20.7)$ & $12(17.6)$ & & \\
\hline Duration of illness, year & $1.3(0.9)$ & $2.2(1.2)$ & $2.5(1.3)$ & & 0.295 \\
\hline \multicolumn{6}{|l|}{ Current diseases(\%) } \\
\hline Cardiovascular disease & $76(49.3)$ & $137(54.6)$ & $35(51.5)$ & & \multirow[t]{3}{*}{0.181} \\
\hline Other somatic diseases & $48(31.2)$ & $63(25.1)$ & $15(22.1)$ & & \\
\hline No somatic diseases & $30(19.5)$ & $51(20.3)$ & $18(26.4)$ & & \\
\hline Number of outpatient visits and & $4.6(1.8)$ & $6.5(2.9)$ & $10.3(3.8)$ & & 9.964 \\
\hline \multicolumn{6}{|l|}{ hospitalizations } \\
\hline GAD-7 score & $6.55(2.52)$ & $8.39(2.41)$ & $12.76(3.28)$ & 9.431 & 0.028 \\
\hline PHQ-9 score & $8.53(2.22)$ & $10.02(2.25)$ & $13.87(3.13)$ & 14.352 & 0.001 \\
\hline
\end{tabular}

Data are expressed as frequency (\%), mean \pm standard deviation. 
PHQ, Patient Health Questionnaire;GAD, General Anxiety Disorder.

Table 4ロThe comparison of anxiety and depression score in patients with different degrees of somatic symptoms

\begin{tabular}{lccccc}
\hline & Mild & Moderate & Severe & F & $P$ \\
\hline Number of patients & 154 & 251 & 68 & & \\
GAD-7 score & $6.55 \pm 2.52$ & $8.39 \pm 2.41$ & $12.76 \pm 3.28$ & 9.431 & 0.028 \\
\hline PHQ-9 score & $8.53 \pm 2.22$ & $10.02 \pm 2.25$ & $13.87 \pm 3.13$ & 14.352 & 0.001 \\
\hline
\end{tabular}

Table 5םLogistic regression analysis of predictive factors of somatic symptoms

\begin{tabular}{|c|c|c|c|c|c|c|}
\hline \multirow[t]{3}{*}{ Variable } & \multirow{3}{*}{$\begin{array}{c}\text { Regression } \\
\text { coefficient }\end{array}$} & \multirow{3}{*}{$\begin{array}{c}\text { Standard } \\
\text { error }\end{array}$} & \multirow{3}{*}{$\begin{array}{l}\text { Wals } \\
\text { value }\end{array}$} & \multirow[t]{3}{*}{ OR } & \multicolumn{2}{|c|}{ 95\%C.I. } \\
\hline & & & & & Lower & Upper \\
\hline & & & & & limit & limit \\
\hline GAD-7 & 1.943 & 0.550 & 13.197 & 6.526 & 2.903 & 13.045 \\
\hline score & & & & & & \\
\hline PHQ-9 & 2.105 & 0.571 & 15.148 & 8.020 & 3.470 & 18.930 \\
\hline score & & & & & & \\
\hline Female & 1.540 & 0.416 & 8.451 & 4.440 & 1.059 & 9.073 \\
\hline constant & 1.463 & 0.947 & 2.385 & 0.232 & & \\
\hline
\end{tabular}

